\title{
Five-Year Plan for Nutrition Research and Training: Executive Summary
}

\author{
NATIONAL INSTITUTE OF CHILD HEALTH AND HUMAN DEVELOPMENT
}

INTRODUCTION. The responsibilities of the National Institute of Child Health and Human Development (NICHD) in nutrition research comprise a variety of biomedical and behavioral areas. These include the nutrient requirements of the pregnant and nursing woman, the fetus, infant, child, and adolescent; nutritional antecedents of disease; assessment of nutritional status at all developmental stages; and the role of nutrition in the treatment of inborn errors of metabolism. To meet these responsibilities better, the NICHD staff tries to keep the Institute's programs current with contemporary science by periodically sponsoring an assessment of research progress in the appropriate areas and analyzing the needs and opportunities for nutrition research.

The last such NICHD-sponsored assessment of nutrition research, called the Nutrition Research Plan, was published 10 years ago. Since then, the nutrition field has entered a new world of opportunities, and there has been a need for an update in our plans. Accordingly, the staff of the Endocrinology, Nutrition and Growth (ENG) branch of the NICHD recently invited a multidisciplinary panel of noted investigators from the ranks of active scientists outside the Institute to describe the state of nutrition science in the relevant areas and to identify promising research questions. These consultants divided their topics into 13 chapters, drafted the chapters, and submitted them to the group for review and discussion. The final product represents a consensus of the group on the chapter contents, statements, and recommendations. Research directions and opportunities described in the chapters, which exceed current budgetary capacity for implementation, were reviewed by staff of the NICHD for feasibility and relevance to the specific mandate of the Institute. At the end of each chapter is a staff response, representing the staff's best judgment on desirable actions for the future, taking into account the Institute's mission and the likely availability of resources.

Members of the National Advisory Child Health and Human Development Council participated in the development of this plan. The document was reviewed by the Planning and Agenda Committee of the Council and endorsed by the entire Council before publication.

The names of the consultants who formulated the plan and a summary of their recommendations and the staff responses are printed below.

Requests for reprints of this summary or for copies of the complete plan may be made to: Office of Research Reporting, National Institute of Child Health and Human Development, Building 31, Room 2A32, Bethesda, MD 20892; phone: 301-496-4757. Other inquiries should be addressed to: Ephraim Y. Levin, M.D., Medical Officer, Endocrinology, Nutrition and Growth Branch, National Institute of Child Health and Human Development, Executive Plaza North Building, Room 637, Bethesda, MD 20892.
Contributors

Lynda C. Bennett
Dennis M. Bier
Saul W. Brusilow
Darla E. Danford
William H. Dietz, Jr.
John D. Fernstrom
Cutberto Garza
Gilman D. Grave
Harry L. Greene

Ekhard E. Ziegler
K. Michael Hambidge William C. Heird James G. Hill

Ephraim Y. Levin Reynaldo Martorell Frank Sacks

John W. Sparks

Louis E. Underwood W. Allen Walker

The science of nutrition has been defined as the sum of the processes concerned with the growth, maintenance, and repair of the living body as a whole or of its constituent parts. Along with organic chemistry, it was the forerunner of modern biochemistry and molecular biology. In recent years, however, there have been profound changes in the perception of nutritional problems and in the methods used to resolve them. Modern mechanistic investigations in molecular biology are expected to lead to explanations of observations in clinical nutrition and provide leads to rational therapies for clinical conditions.

Nutrition research in pregnancy and childhood demands approaches that are noninvasive. Much applied clinical investigation therefore requires extensive preliminary work in model systems, for which the use of transgenic animals offers an exciting new approach. Recent technological advances in the use of stable isotope mass spectrometry, magnetic resonance spectroscopy, and positron emission tomography provide new avenues to minimally invasive studies in children, but require large outlays for equipment. Nutrition departments and individual investigators should make more use of available National Institutes of Health (NIH) grant mechanisms for obtaining access to shareable instrumentation for medical research.

$\mathrm{NIH}$-supported opportunities for training support are also not being used well by the nutrition community, even though the present training rate will not supply the scientists and clinicians needed to implement the recommendations of recent National Academy of Sciences and Department of Health and Human Services reports for research in nutrition.

NICHD responsibility. The Referral Guidelines of the NIH Division of Research Grants lists 15 areas as NICHD responsibility in nutrition research. Each of these areas is addressed in one or more chapters of this plan, which describe scientific areas of need and opportunity. In times of budgetary constraint, it is important for scientists to be aware of these areas and initiate research proposals to address these topics themselves, because only in exceptional circumstances will it be possible for NICHD staff to target many of these needs and opportunities for stimulation by requests for (grant) applications or requests for (contract) proposals. In the future, NICHD staff will need to add to these ways of promoting nutritional research by actions that will cast the Institute more often in the role of initiator or facilitator of activities to be undertaken by the research community itself. 


\section{NUTRITIONAL EPIDEMIOLOGY}

Nutritional problems continue to plague children of indigenous minorities and recent immigrants in the United States. Iron deficiency and other types of malnutrition are not uncommon in inner-city environments and on Indian reservations. Overnutrition and obesity are a problem in most segments of the population, but are more common among certain minority groups. Prevention of obesity and the promotion of healthy eating patterns in childhood should reduce the incidence of cardiovascular disease, hypertension, diabetes, osteoporosis, and possibly other disorders of later life. However, current recommendations for preventing cardiovascular disease in adults may not be appropriate for growing children.

How to identify optimal growth within the genetic potential of individuals is not clear. Nutritional factors powerful enough to markedly depress growth may affect other important functions or even be felt in a subsequent generation; conversely, maximal growth may exact penalties in middle age and beyond. The public health significance of nutritional problems rests on considerations of their prevalence and the degree of functional impairment that they cause.

The nutritional requirements of the pregnant or lactating woman, as measured in terms of fetal or infant outcome, are still uncertain. Moreover, these needs may differ among different ethnic and socioeconomic groups.

Research in all these areas is handicapped by limitations in nutrition data banks, in diet assessment methods, and even in food composition tables.

Possible staff responses to the need for information in nutritional epidemiology include support for studies of anthropologic differences in nutritional status, methods for identifying the childhood antecedents of adult disease, and better methods for nutritional status assessment and the nutrient composition analysis of foods.

\section{MATERNAL-FETAL NUTRITION}

The complicated nature of the prenatal physiologic system, along with ethical considerations, requires that most research on it be descriptive in nature or be performed with animal models. Nevertheless, societal factors and the increased recognition of the importance of prenatal care to neonatal outcome demand better information on optimal nutritional practices in pregnancy. Recent epidemiologic findings suggest an important influence of prenatal nutrition on the incidence of birth defects, and its effect on birth weight is well established.

Routine obstetrical care poses practical nutritional questions about optimal weight gain, the need for nutritional supplements, and the proper nutritional preparation for lactation. Moreover, recent advances in methods for fetal assessment, such as chorionic villus sampling, ultrasonography, and percutaneous fetal blood sampling, suggest the possibility of intrauterine nutritional therapy. Other technologies, such as stable isotope turnover studies using mass spectrometry, magnetic resonance imaging, or doubly labeled water studies of energy balance, could be extremely useful but have been little applied to studies during pregnancy.

Current work that promises to improve the survival of smaller and sicker infants may lead to more long hospitalizations for growth and nutritional management. Because approximation of the intrauterine growth rate is a general criterion for evaluating the progress of low birth weight infants, better information is needed about the variables controlling prenatal growth and the long-term consequences of variations in it.

Possible staff responses to the recommendations in this chapter include encouragement of the development of minimally invasive methods and better animal models for studying nutritional effects on fetal growth and development; sophisticated epidemiologic studies of the effect of nutritional variables on the health of the pregnant and lactating woman and on the fetus and infant; studies of the placenta as an organ of nutrient transport; and facilitation of the use of the methods of molecular biology in studies of fetal growth and metabolism.

\section{MATERNAL-FETAL NUTRITION AND FETAL GROWTH FACTORS}

Studies of the hormonal regulation of fetal growth and how nutrients modulate growth factor secretion and action are difficult because of limited access to the fetus and the complexity of the fetal-placental-maternal system. Research on this system in the near future will need to use postnatal and in vitro models.

There is no accepted general classification for growth factors, which are defined as peptides that regulate cell proliferation and differentiation. Most growth factors are believed to act by binding to specific cell surface receptors, transducing signals across the cell membrane, and triggering a cascade of intracellular events.

Despite its anabolic actions, insulin is probably not a true growth factor, although its role in fuel homeostasis makes it critical to intrauterine development. Its relatively weak stimulation of cell proliferation is probably related to its homology to IGF. Synthesis of IGF occurs in most organs and tissues in intrauterine life, with transcripts of IGF-II far more abundant than those of IGF-I. Whether IGF-I is nutritionally regulated in the fetus as it is postnatally is unknown.

The regulation of IGF, especially IGF-I, by nutrient intake appears to be an important link between nutrition and growth. Dietary calories and protein are both involved in the maintenance of IGF-I levels, which seem to indicate the state of metabolism even better than does nitrogen balance. The effect of diet on growth seems to be mediated at the growth hormone (GH) receptor level for fasting and at the post-GH receptor level for protein deprivation, in either case reducing IGF-I synthesis at a pretranslational level.

The results of most studies on nutritional modulation of IGFI secretion conducted on young postnatal animals will probably hold for the fetus, but some work on in utero systems will still be necessary. General and tissue-specific growth factors and their receptors need to be identified, which will require highly sensitive and specific assays. Definitive studies of the nutritional regulation of growth factors and growth in the fetus must be preceded by the development of animal model systems in which nutrients can be manipulated.

A variety of other growth factors have effects on the replication and development of embryonic or fetal tissues, which makes it. likely that they have important roles in development. These include nerve growth factor, epidermal growth factor, transforming growth factors- $\alpha$ and $-\beta$, heparin-binding growth factors, and platelet-derived growth factors. The role of nutrition in their regulation is not known. Other substances with growth-promoting actions more specific than any of the above are vasopressin, bombesin, colony-stimulating factors, lymphokines, erythropoietin, and the protein products of cellular protooncogenes.

Possible staff responses to the recommendations in this chapter include support for the development of better animal models for study of the nutritional control of fetal growth and of sensitive quantitative assays for growth factors and their receptors. These are necessary for studies of the effect of in utero malnutrition on growth and development and on the role of growth factors in the gestational abnormalities resulting from maternal undernutrition, overnutrition, diabetes, hypertension, and substance abuse.

\section{HUMAN MILK STUDIES}

Although an increase in the frequency and duration of breastfeeding is among the health goals developed by the Surgeon General, a lack of understanding of the determinants of breastfeeding impedes the development of strategies for effecting a change in current infant feeding practices. Among the influences that are recognized are the age, education, ethnic group, socio- 
economic status, religion, and employment of the mother and her partner. These factors may interact with the health system that supports the mother-infant dyad and with maternal anthropometric characteristics, health and reproductive histories, lactation management knowledge, sociocultural value's, and diet.

Synthesis and secretion of milk. Mechanisms that control the concentration of specific nutrients in milk, especially fat, are poorly understood. The same is true for the constellation of immune factors in milk. Moreover, it is important to move beyond simple quantitation of immune components to appraisals of their protective efficacy. Efforts being directed at the "humanization" of artificial formulas are difficult to evaluate because of limited understanding of the significance of variables in physical properties of milk, such as the compartmentalization of constituents, the interdependence among constituents for functional activity, and the physiologic significance of these considerations for the infant. Finally, the infant's responses may have a role in modulating the composition of milk just as they regulate the volume.

Infant outcomes. Breast-fed and bottle-fed infants differ in incidence of gastrointestinal morbidity, intake of energy, and growth patterns. Breast-fed infants also seem to have lower minimal energy expenditure, heart rate, and core temperature. Moreover, feeding of breast milk affects the immune system, not only by direct-acting antimicrobial factors but also through the presence of antiinflammatory agents and factors that speed the maturation of immunologic functions. Finally, infant behavior may be influenced by feeding mode because of either different components of the milks, different amounts of nutrients consumed, or differences in feeding practices.

Little work has been done on the role of human milk in the nutritional management of ill infants. Nevertheless, the frequency and severity of gastrointestinal infections have been found to be less among breast-fed infants, with the barrier role of secretory IgA usually cited as the likely mechanism. In other situations in which breast milk may protect against infection, it is not clear whether it induces changes in the infant that enhance responses to stress or if specific components of human milk lessen the virulence of offending agents.

The available data suggest that breast-feeding also decreases the incidence and severity of respiratory infections. The evidence for an effect on the development of chronic diseases is less convincing but suggestive. Some viruses may be transmitted from the mother to the infant via the milk, but (except for human immunodeficiency virus) these have not been shown to cause disease. In contrast, there is preliminary epidemiologic evidence that the risks of the later development of juvenile diabetes mellitus, lymphomas, and Crohn's disease are less in breast-fed infants. Because breast-fed infants have a different growth pattern than bottle-fed infants, growing faster in the first few months of life and more slowly after that, the first-year growth pattern rather than the nature of the feed could be the variable correlating with later predisposition to chronic disease.

Maternal outcomes. Few studies have concentrated on the effect of lactation on the mother's health. The physiologic stress of lactation is significant, the mother transferring more nutrients to the nursling during the first 5 months of exclusive breastfeeding than she provided to the fetus in the 9 months of pregnancy. Basic information is available regarding the endocrine changes in lactation, but the relationship between these and other systemic functions is undefined.

The longer-term consequences of lactation on maternal health are also unknown, especially among women with other nutritional risks. Areas warranting investigation include the permanence of metabolic consequences of lactation that may influence health, changes in calcium metabolism, the predisposition to specific cancers, and effects on maternal self-image.

Possible staff responses to the need for research on human milk include the development of studies of the short- and longterm effects on the infant of specific components of human milk and the process of breast-feeding; the effects of nursing on the mother's physical and mental well-being; and the factors in maternal diet and infant behavior that control the composition of milk.

\section{FEEDING OF TERM INFANTS}

Although exclusive breast-feeding for the first few months of life is recommended for all normal term infants, there are still unanswered questions about other aspects of infant nutrition.

The 0- to 6-month old infant. The magnitude and mechanism of the advantages of breast-feeding remain to be defined. These include the apparent protection against infection, the stimulation of maturation of the gastrointestinal system and the CNS, and the enhancement of nutrient absorption. The minimum duration of breast-feeding needed to provide maximum nutritional benefits is an important question; if breast-feeding provides significant advantages in a developed society, this issue has social policy implications with respect to the duration of maternity leave, the need for work-site day-care facilities, and the encouragement of nursing.

Studies of the effects of breast-feeding and bottle-feeding have other potential values. A better understanding of the differences in rate and composition of weight gain in the bottle-fed and breast-fed infants could help our understanding of the mechanisms controlling growth. The reason for their apparent difference in intakes could clarify the role of "imprinting" of intake patterns in infancy on long-term eating patterns, especially with respect to energy, cholesterol, and salt intake.

The 6-to 12-month-old infant. It is necessary to learn when and why exclusive breast-feeding becomes nutritionally inadequate to make recommendations for the introduction of supplemental feeding. Obtaining this information will require assessment of the infant's nutritional status, for which methods are rudimentary. Few studies have addressed the nutritional needs of this age group, which must be estimated by extrapolations from data obtained on younger or older children. Requirements for iron, fluoride, zinc, and most of the vitamins are particularly uncertain.

A large percentage of infants 6 to 12 mo of age are fed bovine milk; many of them in this country are given low-fat milk. They are thus receiving protein and sodium intakes far in excess of safe and desirable levels, but only about one third as much of the essential lipid linoleic acid. The question of deleterious effects of this practice has economic implications for the individual family as well as for society, which subsidizes the feeding of infants through a variety of agencies, because cow milk is so much less costly than infant formula.

Another important question concerns the relationship of the intake of nutrients during early life to the development of later disease, particularly allergic disorders, osteoporosis, hypertension, and atherosclerosis. For long-term effects, large-scale epidemiologic studies may be required. Following individuals from infancy to adulthood poses practical difficulties, but much can be learned from follow-up studies of shorter duration. For example, if diet in infancy correlates with serum lipid values at age five and serum lipid levels in childhood and adolescence correlate with risk factors for atherosclerosis in adult life, an impact of lipid intake during infancy on the development of atherosclerosis would be suggested. Well-designed retrospective studies would also be valuable in assessing the effect of early diet on later degenerative disease.

The sick infant. The effect of illness or other stress on the energy needs and other nutrient requirements of infants has not been established. Equally important questions concern the effects of nutrients on the progression of chronic disease states. Is the growth failure of chronic illness an undesirable side effect of nutrient diversion, or is it an adaptive mechanism to reduce cardiac, pulmonary, or renal metabolic demands?

Parenteral nutrition has a crucial role in the management of 
sick infants, but much remains to be learned about it; for example, the retention of nitrogen is generally less with parenteral amino acid mixtures than with enterally delivered regimens. The metabolism of some amino acids may differ with different routes of administration, and some amino acids that are nonessential for older children are essential for the newborn. The optimal combination of glucose, which is more effective in promoting nitrogen utilization, and lipids, which provide more calories per mole while producing less $\mathrm{CO}_{2}$, should be determined. Infants may have difficulty clearing i.v. fat emulsions, possibly because of carnitine deficiency. Finally, the ability of infants to elongate and desaturate both n-3 and n- 6 fatty acids needs to be determined, inasmuch as neither enteral nor parenteral formulas contain elongated and desaturated members of these fatty acid families.

Possible staff responses to the recommendations in this chapter overlap somewhat with those in the chapters on human milk and intestinal maturation. Particularly intriguing is the novel idea that overlapping tracking studies of diet and risk factors might provide a practical alternative to impossibly long followup study designs. It seems important to learn why breast-fed and bottle-fed infants differ in their rates of weight gain and what the significance is of these differences.

\section{NUTRITION AND THE PRETERM INFANT}

The newborn preterm infant. Because of life-threatening organ system disorders in the first few days of life and formidable obstacles to nutrient administration by the gastrointestinal and i.v. routes, adequate nutritional management of low birth weight infants is often postponed for days or weeks. By the time complete nutritional regimens are instituted, a substantial negative balance of energy and most nutrients has been incurred, and weight gain typically does not occur until the infant is several weeks old. Not only does this period of partial starvation prolong hospitalization, but it also may have short- and long-term adverse consequences, such as the well-known adverse effects of malnutrition on humoral and cellular immunity.

Thus, it might seem that institution of full parenteral nutrition immediately after birth would be desirable. The feasibility of this has not been tested; currently, only glucose and electrolytes are provided in the first few days, and the glucose intolerance typically present limits the energy input from this source to less than expenditure. Because the preterm infant has little body fat, the energy deficit results in protein catabolism. The mechanism of the glucose intolerance needs to be elucidated so strategies for overcoming it can be developed, and the usefulness of other fuel substrates and amino acid mixtures should be studied. Prevention of the long-term complications of parenteral nutrition such as cholestasis and metabolic bone disease is an additional necessary research focus.

The practice of delaying enteral feedings is a result of the fear of inducing necrotizing enterocolitis (NEC), even though clinical trials have not supported this practice. Prevention of NEC, a potentially devastating condition, requires a better understanding of its etiology and pathogenesis. The possible protective effects of minimal enteral feedings, especially with the inclusion of nonnutritive substances such as immune globulins, colostrum, and hormonal components of breast milk, need to be studied. Immature intestinal function, particularly motility, is an additional obstacle to enteral nutrition that warrants attention.

The growing infant. Suboptimal nutrition can lead to slow growth rates and altered body composition, such as an increase of body fat. Delayed behavioral development and prolongation of the hospital stay have medical and social implications, and undermineralization of bone carries a risk of fractures. The major significance of delayed growth, however, is the potential for longterm consequences. There is thus a need to define minimal requirements and optimal intakes with accuracy.

Better methods for the monitoring of nutritional status and the selective adjustment of nutrient intake might substitute for a detailed definition of changes with growth and age and the range of individual variation.

Nutrition and disease. Hypercatabolism induced by the stress of intercurrent illness in preterm infants affects the requirement for calories and probably for specific nutrients as well. Extrapolations of needs from studies of older subjects may not be valid. In bronchopulmonary dysplasia (BPD), the increased work of breathing increases energy requirements, accounting for most of the associated growth failure. A compensatory increase in energy intake is often impossible to manage in BPD because of the concurrent need to restrict fluid intake. Similarly, an increased intake of calcium and phosphorous is often avoided in metabolic bone disease because of a fear of nephrocalcinosis.

Human milk. The provision of mother's milk to the premature infant is thought to provide nonnutritional benefits and to give the mother a sense of linkage to the infant that may counteract the disruption of bonding caused by the infant's extended hospitalization. But lactation is more difficult to establish after premature birth, and the content of some nutrients in human milk is insufficient for the premature infant, making supplementation necessary. Most of the presumed benefits of human milk (such as its role in the prevention of NEC) are not documented, and prospective, controlled, randomized studies cannot be expected. Nevertheless, the possible effects of milk constituents on gut development, local and humoral immunity, and protection against infection warrant study.

Possible staff responses to the recommendations in this chapter include studies of more effective and safe mixtures for the early initiation of complete parenteral nutrition in premature infants, the role of specific enteral nutrients in the maturation of their organ systems, and methods for noninvasive evaluation of their nutritional status. Investigations of the pathogenetic mechanisms of NEC are important to progress in this area.

\section{NUTRITION AND GASTROINTESTINAL DEVELOPMENT}

Role of breast milk. The neonatal intestine is immature and needs to grow and differentiate for its digestive, immune, and environmental barrier functions. Limited in vitro studies have demonstrated effects of polypeptide growth factors secreted in human milk on cell proliferation and differentiation, but no in vivo studies have defined their effects on intestinal maturation. Breast milk seems to increase secretory IgA in mucosal secretions and stimulate proliferation of B cells. The B cells present in milk may have effects on immune system maturation. The efficacy of breast-feeding in reducing atopic disease is still unclear, despite many studies.

The role of lactose, specific growth factors, and neuropeptide/ enteric hormones in the development of gastrointestinal motility and epithelial development should be an important area of study over the next 5 years. Approaches based on animal model systems or enteric organ culture of fetal, premature, or neonatal biopsy material should be used.

Diet-enterocyte interactions. Environmental factors, including breast milk substances, react with tissue receptors to affect the development of enterocyte function. Nutrient interactions can modify the developmental programming of gene expression and the development of intestinal host defenses. These influences need to be defined at molecular, genetic, enzymatic, and cellular levels.

The immaturity of the neonatal gut is apparently a factor in NEC, infectious diarrhea, and allergic disorders, and possibly in inflammatory bowel disease and celiac disease. The complex evolution of the bacterial milieu in the infant intestine during the changes from the sterile in utero environment to the contaminated world and from maternal milk to solid food affects the development of intestinal mucosal structure and function; conversely, host factors influence the nature of the indigenous flora. A determination of the effect of nutrients on the expression of 
disease in the perinatal period will require multicenter prospective controlled studies.

Possible staff responses to the recommendations in this chapter include stimulation of research on the effects of specific components of human milk on intestinal motility and the maturation of immunologic systems. Animal or organ culture systems could be developed for such research. Basic studies are indicated to elucidate how intestinal epithelium is regulated by the external environment, including mechanisms of signal transduction, and clinical studies to clarify how this regulation is deranged in disease states like NEC.

\section{NUTRITION AND NERVOUS SYSTEM FUNCTION}

Chronic nutrient deficiencies during development can have lasting effects on the brain. Protein and protein-calorie malnutrition during late gestation and early postnatal life can produce specific neurotransmitter deficits and defects in glial cell proliferation, myelination, and the development of neural populations. Deficiencies in essential fatty acids, which are important in the formation of neural membranes, have been shown to have functional consequences in experimental animals. For example, $\alpha$-linolenic acid deficiency during fetal development results in visual defects and (possibly secondary) learning deficiencies. More subtle but functionally significant effects could include the alteration in neural membrane composition and the synthesis of acetylcholine produced by a diet high in n-6 fatty acids.

Dietary deficiencies of vitamins also produce notable CNS effects. Niacin and thiamine deficiencies produce neurologic deficits in animals and humans, and pyridoxine deficiency can reduce CNS levels of $\gamma$-aminobutyric acid and increase seizure susceptibility. Variations in dietary choline influence the synthesis of acetylcholine, with possible functional consequences.

Iron deficiency produces behavioral abnormalities in animals and children, with (possibly secondary) learning deficits in the latter. Most CNS iron is in lipids, including myelin, but in neurons the highest concentrations are in the substantia nigra, where it may account for lipid peroxidation and neuronal toxicity in Parkinsonism.

Iodine deficiency in pregnancy interferes with thyroid hormone synthesis, producing subsequently profound and irreversible CNS developmental abnormalities postnatally. Excessive intake of manganese and lead produce dementia, and zinc deficiency during development causes brain malformations. For none of these effects is the mechanism known.

Acute changes in intake also can cause alterations in the CNS. Single-meal ingestions of tryptophan or tyrosine can alter their uptake into the brain and influence the production of serotonin or cathecholamines, respectively.

Nutritional research on the brain should include studies of other factors that influence the access of nutrients to the CNS, such as changes in the blood-brain barrier, and of adaptation of the brain to the use of different energy sources. It includes work on nutritional effects on the peripheral nervous system (PNS) as well as the CNS. Peripheral nerve axons are separated from the circulation by a blood-nerve barrier with nutrient transport mechanisms like those at the blood-brain barrier, but the nerve terminals are not; thus, nutrient access to the terminal may differ from that to the axon.

Behavioral scientists have investigated the CNS mechanisms that determine nutrient selection. Little of this work has been done in children, but it is known that children can modulate their caloric intake in response to the caloric density of the food consumed and show sensory-specific satiety for foods just consumed. Cultural factors can distort such regulatory mechanisms, however, and influence lifelong patterns of food selection.

Research on nervous system development is of particular relevance to NICHD responsibilities. At each stage of development, we need to understand in both CNS and PNS the nutritional demands of the dividing neurons, the dendrites and ex- tending axons, each glial cell type during its proliferative and myelination phases, and the ependymal cells. The ultimate utility of such knowledge will be to identify key components of the maternal diet for proper nervous system development in utero and for the optimal composition of breast milk for postnatal nervous system development; to determine the appropriate components of enteral and parenteral formulations for premature infants; and to aid the formulation of properly constituted diets for children afflicted with metabolic diseases. We need to know what nutritional vulnerabilities exist in childhood and adolescence, and how nutrition interacts with the nervous system to influence the onset of puberty. Although animal studies can provide much preliminary information, for confirmatory studies in humans there is a need for better noninvasive methods for examining nervous system biochemistry.

There are few robust training programs in this field; the quality of work would benefit enormously by more training of individuals in both nutrition/metabolism and appropriate areas of neuroscience.

Possible staff responses to the recommendations in this chapter include efforts to stimulate research on both the effects of nutrients on CNS development and maturation and the effects of the maturing CNS on the development of food intake behavior. Studies of transport across the blood-brain and blood-peripheral nerve barriers should proceed at the molecular level, along with studies of the mechanisms of nutrient control of CNS and PNS maturation. Studies of optimal nutrition of different nervous system cell types will require support for both in vitro and noninvasive in vivo research. Support could also be strengthened for whole-animal studies of factors involved in establishing various eating behaviors. To facilitate research in all these areas, the Institute might encourage training of individuals in both nutrition/metabolism and neuroscience.

\section{VITAMINS IN INFANCY AND CHILDHOOD}

Increasing use of long-term total parenteral nutrition (TPN) in pediatrics has highlighted the need for more knowledge of vitamin requirements, blood and tissue levels, and potential adverse effects. For better evaluation of vitamin nutriture, there is a pressing need for more reliable micromethods of assay of vitamers and their metabolites.

Water-soluble vitamins. The gastrointestinal tract and liver modify orally ingested vitamins for use as cofactors in enzymatic reactions. Because they are little stored, water-soluble vitamins must be administered daily, but can be supplied in doses in excess of expected needs without much danger of toxicity. Requirements may differ for enteral and parenteral administration because different fractions of the dose encounter first the liver or kidneys. The relative intake of vitamins by infants receiving TPN generally exceeds that from formulas by several-fold and that from human milk by 10 -fold.

Toxicity from water-soluble vitamins may occur from massive doses or impaired urinary excretion, especially in premature or sick children. Parenteral doses should be adjusted appropriately in low birth weight infants.

Thiamin requirements depend on carbohydrate intake, but the quantitative relationship is not known. The currently used screening method for thiamin deficiency does not detect thiamin excess, and more definitive measures of thiamin and its metabolites are needed.

Riboflavin inactivation by light can lead to deficiency in infants receiving marginal amounts, but currently recommended doses are probably excessive, especially for preterm infants, leading to photohemolysis. Recently described methods for quantitating serum and red cell riboflavin and cofactors derived from it should provide the basis for more definitive studies of their metabolism.

Vitamin $B_{6}$ is extensively involved in amino acid metabolism, so its requirement is related to protein intake. This relationship 
has been little studied in children, especially those on TPN. To measure i.v. needs, clinical studies with controlled amino acid intake and measurements of the cofactor derivatives of $B_{6}$ are needed.

Niacin in the diet is supplemented by niacin derived from dietary tryptophan, but the quantitative value of this supplementation is unknown. Data on the niacin requirement in childhood are unavailable, and currently used assay methods are cumbersome.

Folate deficiency is one of the most common hypovitaminoses. Because of this and because information on alterations in folate metabolism in disease states in childhood is lacking, future studies of this vitamin are indicated.

Cobalamin $\left(B_{12}\right)$ stores greatly exceed daily needs in healthy children on a normal diet, but deficiency may develop in the breast-fed infants of strict vegetarians and in children with small bowel disease. Studies to determine the most appropriate intake in premature or diseased infants are also needed.

Lipid-soluble vitamins. These substances must be made soluble if they are to be administered intravenously or in an aqueous oral suspension. Polysorbate, a synthetic detergent, has long been used for this purpose, but circumstantial evidence of its toxicity to preterm infants indicates a need to document its safety, especially when used in TPN. A second problem with lipidsoluble vitamins is the potential for toxic overdose; the dose ranges for safety and efficacy in preterm infants are undefined.

In adults, vitamin A administered intravenously is stored as retinyl palmitate until a regulated hydrolysis liberates retinol, but no such process has been shown in children. Moreover, retinol is lost from TPN solutions by photodegradation and by adherence to the plastic tubing, a particular problem in the management of very low birth weight infants because of the small volumes of TPN solutions administered to them and the high light intensity in nurseries. Growing children have only small stores of retinol and preterm infants have virtually none. A new method of retinol and retinyl palmitate determination by HPLC could be used to follow levels in blood and tissues. Investigation of the relationship between vitamin A levels and BPD is a high priority.

Vitamin E comprises several forms of tocopherol that function as biologic antioxidants. Deficiency of vitamin E, which increases red cell hemolysis and can result in progressive neuronal damage, is a feature of chronic fat malabsorption. Although large doses of $E$ have been suggested for prevention of complications of prematurity and diseases associated with peroxidative injury, no study has clearly demonstrated a therapeutic role for it in pharmacologic doses.

Newborn infants have low prothrombin levels and lack the intestinal flora that might synthesize vitamin $\mathrm{K}$, so they are usually given an intramuscular injection of $\mathrm{K}$ to forestall intracranial hemorrhage; human milk contains little vitamin $\mathrm{K}$. Toxicity from parenterally administered emulsified $\mathrm{K}$ is rare, except for large doses. Recently developed HPLC methods for vitamin $\mathrm{K}$ analysis could allow better definition of dietary needs.

Vitamin D is involved in the regulation of cellular differentiation, as well as in calcium and phosphorus metabolism. Studies of its metabolism during early development in very low birth weight infants would be important.

Developmental and adaptive changes. Because of rapid growth, vitamin needs are probably highest in the first 6 months of life. Growth is less rapid during the second 6 months, but recommended daily allowances (RDA) change little. In U.S. infants, consumption of most vitamins varies from $100 \%$ to $350 \%$ of the RDA. Prematurity, disease, drug-vitamin interactions, or extremes of activity may alter vitamin absorption, catabolism, or excretion, changing the dosage needed for body saturation.

Recommendations for future studies. Estimates of dietary requirements for vitamins in childhood are based on extrapolations from the intake of thriving breast-fed infants. Only a few studies have correlated blood or urine levels with intakes. Recommen- dation for i.v. dosage are not based on much data from children, but some studies suggest that recommendations for preterm infants should differ from those for term infants.

Vitamins A, D, and E have storage depots, and toxicities from their overdosage are well recognized. There is a need for careful pharmacokinetic studies of vitamin metabolism in infants and children, not only under normal conditions in different stages of development but also in various disease states. Finally, the nutritional role of substances "conditionally essential" under adverse metabolic conditions needs investigation.

Possible staff responses to the recommendations in this chapter include soliciting research proposals for the development of methods for evaluating vitamin status in children and applying these methods to studies of the metabolism of compounds administered by different routes to small babies and sick children and examining the role of vitamins as therapeutic agents in specific pathophysiologic states.

\section{TRACE ELEMENTS}

Ten elements are known to be essential micronutrients in human nutrition. Six others have demonstrated or suggested roles in other mammalian species, and another three possibly have roles. There is growing evidence of the practical importance of microelements in infancy, childhood, and the reproductive cycle.

Molecular and cellular biology. The methods of molecular biology provide the tools for understanding at the molecular and cellular levels the role of trace elements in fetal and postnatal development and the mechanisms of placental transport and intestinal absorption. Kinetic studies with stable isotopes should shed light on how trace element deficiencies occur despite significant adaptive mechanisms. Identification of the genetic defects in certain inborn errors of metabolism should clarify the molecular basis of intestinal absorption. Trace metal interactions, especially among iron, zinc, and copper, are of special concern.

Deficiencies and imbalances. The clinical importance of deficiencies in iron and iodine intakes during growth stages of the life cycle is well known, but there are gaps in our knowledge. Mild iron deficiency, even without anemia, can have adverse effects on cognitive development in the young child, and mild maternal iodine deficiency can cause subtle injury to the fetal brain even without overt cretinism. The potential importance of other trace nutrient deficiencies during fetal and neonatal development has been demonstrated in animal models.

The development of better tools for assessing trace element nutritional status, especially relatively simple ones that can be applied to large scale epidemiologic studies, must be a major priority. These should be designed to detect not only overt clinical effects but also more subtle disturbances of function of the immune, endocrine, and nervous systems.

Prenatal study of maternal trace element status, especially with regard to zinc, is an area warranting attention. Evidence suggests that zinc deficiency may contribute to pregnancy-induced hypertension, low birth weight, neural tube defects, or immune system abnormalities. Large-scale intervention trials may be justified in this area. Information is also needed about copper status during pregnancy, inasmuch as experimental mild copper insufficiency during the reproductive cycle and in young animals produces lesions resembling early arteriosclerosis.

Postnatal trace element deficiencies also merit priority. Very low birth weight infants, especially those with BPD, apparently have low selenium status. The incidence, causes, and effects of zinc deficiency also require study in this population, and in term infants as well, inasmuch as there is evidence for a growthlimiting zinc deficiency in some otherwise normal infants and children. Research on the optimum means of supplying dietary iron is also relevant to both the older premature infant and the normal toddler.

Requirements. Better estimates of physiologic requirements as 
well as dietary requirements are necessary for an understanding of the etiology and epidemiology of trace metal deficiencies and the means of their prevention. Interactions among trace elements are among the factors that can affect absorption: Quantitative kinetic studies in humans are required, in addition to studies at the cellular and molecular levels.

Advances in analytical methods such as mass spectrometry have begun to make possible in vivo human studies of requirements. The small number and size of samples available from infants make support for development of additional methods essential. Low body stores, immaturity of the gastrointestinal tract, and rapid rates of postnatal growth contribute to a special concern about requirements for very low birth weight infants.

Fractional absorption of trace elements from human milk seems to be especially favorable; priority should be given to determining what components of the milk are responsible for this.

Childhood requirements need to be defined in relation to growth stage. Chronic diseases may pose special requirements by changing absorption, metabolism, or excretion. Poor bioavailability of nutrients in diets therapeutic for inborn metabolic diseases may exaggerate requirements. Also, acute changes in trace element metabolism, as in the hypermetabolic intensive care patient, seem sometimes to have effects on requirements of substantial clinical importance. Finally, many practical questions remain about the i.v. administration of trace elements, such as the level of the requirements for them and the potentially adverse effects of introducing pro-oxidant metals not tightly bound to their physiologic carrier proteins.

Intakes of some trace elements during pregnancy and lactation are substantially below currently estimated requirements. Research is needed to define these requirements better to determine whether supplements are necessary to prevent clinically significant deficiencies.

Possible staff responses to recommendations in this chapter include developing innovative, minimally invasive methods for assessing trace element status in vivo, preferably in terms of critical cellular functions, and providing more research training in their use. These methods should be useful in determining what large-scale supplementation trials should be attempted and in devising more bioavailable trace element preparations for i.v. use. Basic studies of the mechanisms by which trace elements control fetal and neonatal development and the molecular biology of their transport in the placenta and gastrointestinal tract could also be supported.

\section{CHILDHOOD OBESITY}

The increasing prevalence of obesity in this country is a major public health problem because obesity is a risk factor for degenerative and neoplastic diseases. Morbidity in childhood is rare, but obesity in childhood after the age of four predicts adult obesity with an accuracy that increases with age. The causes of childhood obesity are not necessarily identical to the reasons for its persistence; both are complex and may derive from host or environmental factors.

Energy balance. Obesity results from an imbalance between energy intake and expenditure. The host factors that control energy intake comprise both central (hypothalamic) and peripheral (endocrine and gastrointestinal) mechanisms. In addition, a variety of environmental factors, including geography, socioeconomic class, and television viewing, have been associated with obesity in childhood, but the behavioral alterations that link these factors with obesity remain unspecified. Thus, it is not possible to identify specific behaviors for prevention or alteration. By use of the doubly labeled water techniques and large modern calorimetry chambers, it has been learned that the resting metabolic rate is familial and that low activity levels in infancy may predispose to weight gain, but the relationship of activity to food intake has not been studied in children.
In the promotion of energy storage, host factors such as variations in adipose tissue metabolism are more numerous than environmental ones. Diet composition may have some effect, inasmuch as fat is more likely than carbohydrate to be stored when consumed in excess.

Adipose tissue factors that relate to energy use are much like those relating to storage, but our understanding of them in children is limited. Whether differences in response to lipolytic agents play a role in the persistence of obesity is unresolved. Central (abdominal) fat distribution in children has not been shown to be associated with morbidity as it is in adults. Central fat increases in adolescence, so this developmental stage may pose particular risks for the sequelae of obesity. Whether genetic predisposition to fatness or genetic determinants of fat distribution is the more important factor in morbidity is a critical question.

Therapy. Behavioral and ethnic risk factors for severe obesity have not been characterized well enough to suggest a focus for therapy or indicate its urgency. Fat loss is promoted by strategies that preserve fat-free mass, because basal metabolic rate is thereby maintained. Behavior modification that incorporates exercise and a family component seems most successful of the therapies tried so far. Risk factors for the persistence of childhood obesity and the appearance of its sequelae must be identified in connection with the need to identify developmentally tolerant approaches to weight reduction. Most programs have focused on preadolescents; they should be tested in other age groups as well. The risk of inducing anorexia or bulimia by therapy for mild obesity needs to be evaluated. Finally, work on the safety of extremely restricted diets for severely affected children is essential.

In summary, although altered regulation of energy intake by host or environmental influences plays a major role in the onset and probably the persistence of obesity, the factors that determine satiety should be accorded as much attention as the factors that regulate intake. Host alterations in energy storage may also account for a genetic predisposition to the disease and play a role in its persistence; fat intake is probably the major environmental factor. For energy expenditure, environmental effects are probably a major influence on the origin and persistence of obesity.

Prevention of obesity may be more cost-effective than treatment, so the identification of susceptibility factors could be most useful. In the long term, detailed maps of the human genome may allow identification of genetic subtypes of predisposition to obesity. For substantial progress to be made in prevention and treatment of obesity, more trained investigators and centers for pediatric obesity research must be developed.

Possible staff responses to the recommendations in this chapter include support for investigations of the relative significance of genetic and environmental factors in the development of childhood obesity, biochemical and behavioral markers that predict childhood obesity, the relationship of different types of childhood obesity to the high-risk adult body habitus; and the long-term efficacy of methods for prevention and treatment of childhood obesity. The control of appetite and satiety need investigation at the basic level.

\section{LIPOPROTIENS AND CHILDHOOD ANTECEDENTS OF ADULT CARDIOVASCULAR DISEASE}

Blood cholesterol levels predict the later incidence of cardiovascular disease, the leading cause of death in the United States. Decreasing hypercholesterolemia by medication that lowers LDL cholesterol decreases the incidence of coronary artery disease. Many expert groups have recommended that widespread screening for elevated cholesterol levels be undertaken and a national effort be made to lower them. With some of the suggested approaches, the majority of American adults would receive cholesterol-lowering treatment.

The Committee on Nutrition of the American Academy of 
Pediatrics has been concerned that lowering the intake of fat by younger children might limit the energy intake necessary for adequate growth. In addition, this group has expressed uncertainty about the causal relationship between cholesterol levels in children and adult atherosclerosis.

Consensus among experts as to the proper screening and treatment practices for children would be aided by more knowledge of pediatric antecedents of adult cardiovascular disease.

Antenatal and postnatal influences on blood lipids (imprinting). Blood cholesterol varies in a complex way during gestation, being highest in the first semester, showing a secondary peak at the beginning of the second semester, and then declining gradually to term. Variations in blood cholesterol at birth seem to result from differences in placental metabolism as well as in genetics.

It is not known whether antepartum factors have a lasting influence on lipoprotein levels. However, a link between poor nutrition in early life and later atherosclerosis has been suggested by investigators in Great Britain who found that regions with high maternal and infant mortality in the early 1900 s experienced a high incidence of ischemic heart disease and stroke 40 to 50 years later. A definitive study of the effect of intrauterine growth retardation on atherosclerosis would be impractically complex to control and unfeasibly long term. A more practical objective would be a carefully controlled study of whether the risk factors for cardiovascular disease are different over a 2-year follow-up in normal and small infants.

Infant formula has less cholesterol and a higher proportion of linoleic acid than does breast milk. As a result, formula-feeding produces a lower serum cholesterol than breast-feeding. Most human studies have not found a long-term effect of feeding type on plasma lipids, although two studies found an effect on cholesterol; studies in different species have yielded conflicting results. A new cohort study of human infant feeding, with a 2 -year follow-up of plasma lipoproteins, would be desirable.

Some animal experiments have suggested that the administration of bile-sequestering resins in infancy may produce a longlasting improvement in serum cholesterol, a finding of immense implications if it applies to humans. This could be tested in a double-blind study of infants with plasma LDL levels above the 95th percentile.

Age-related changes in lipoprotein metabolism. The lipoprotein system of the neonate is different from that of later life, with much of the plasma cholesterol in HDL rather than LDL. During the first month of life, HDL cholesterol increases 2-fold, but VLDL and LDL increase even more. The mechanism for this transition is of great theoretical interest. New techniques that allow studies of apolipoprotein metabolism in vivo with nonradioactive tracers could be used to study this problem in neonates.

Another change in plasma lipoprotein concentrations takes place during the period of sexual maturation. Before puberty, LDL cholesterol levels in girls are similar to those in boys, whereas HDL levels are lower in girls than boys. During adolescence, these differences reverse. These changes have many possible causes that should be dissected out, such as rapid growth in physical size, changes in amount and location of body fat, increases in reproductive hormones, and the initiation of aspects of adult life-style such as smoking and alcohol ingestion. Lipoprotein metabolism could be studied experimentally in children with hypogonadism or precocious puberty who are being treated with gonadotropins or gonadotropin weak agonists to identify precisely the hormonal contribution to pubertal changes in lipoproteins.

Differences in dietary responsiveness and risk factors. In both children and adults, considerable individual variation occurs in serum lipoprotein responses to a controlled diet. Twin studies could determine the genetic contribution to this variation. Differences in dietary responses between sexes and among different age groups and races have not been investigated. Racial and socioeconomic differences seem to begin in childhood, but more multiracial studies are needed. No study has satisfactorily ad- dressed the relationships among energy and fat intake, lipoproteins, and growth and development.

Screening for hyperlipidemia. The strategy of screening children at risk for hypercholesterolemia on the basis of data in the family history is being rigorously evaluated. The evidence so far does not indicate that this approach would have important advantages over universal screening. Between one third and two thirds of children with hypercholesterolemia would not receive screening under various high-risk strategies.

Atherosclerosis in childhood. Autopsy studies of American children who die accidentally indicate that atherosclerosis begins in the second decade of life. Perhaps not all of the fatty streaks that are commonly present at this age progress to symptomatic lesions, but such a progression has been demonstrated in dietinduced atherosclerosis in nonhuman primates. A method for noninvasive quantitation of carotid or femoral arteriosclerosis could expand the scope of these studies and allow longitudinal evaluation of the influence of plasma lipoproteins and other risk factors.

Interventions in children. An NIH-supported study of dietary therapy in children with elevated LDL levels is in early stages. Another NIH-funded multicenter study is testing an elementary school-based program of dietary change, physical activity, and abstinence from smoking. However, it is not established that lowering serum cholesterol in children will delay the onset of adult cardiovascular disease, and it is not likely that a randomized trial of this approach could be conducted. A noninvasive measure of atherosclerosis could allow interventions to be tested on the disease process rather than on risk variables.

Possible staff responses to the recommendations in this chapter should not include large-scale intervention trials. Studies of the effects on cholesterol metabolism of variables peculiar to childhood, such as birth weight, manner of infant feeding, later dietary intake, and early treatment with cholestyramine, would be appropriate. A method for the noninvasive measurement of the progression of arterial fatty streaks to more advanced lesions would be extremely powerful. At the basic level, the Institute could encourage work of the dietary and hormonal factors influencing the changes in lipoprotein metabolism that occur at birth and at puberty.

\section{INBORN ERRORS OF METABOLISM AND NUTRITION}

Nutritional therapies are still the mainstay of management for inborn errors of metabolism. Nevertheless, for some disorders no satisfactory dietary treatment has been devised; for some, the methods used ameliorate the most serious pathology but do not optimize outcome; and, for some, the treatment is malodorous or unpalatable. There is often retarded growth or secondary dietary deficiencies.

Pathophysiologic issues. For most genetic diseases, the pathophysiology is unknown. An understanding of how the biochemical abnormalities produce the clinical pathology could lead to more rational therapy. Within each inborn error of metabolism, however, there is likely to be genetic heterogeneity. Analysis of genotypic differences by molecular genetic methods could indicate which biochemical variables lead to individual differences in response to therapy within the same phenotype. In addition, sometimes a phenotypically normal, heterozygous female carrier of a mutant allele accumulates toxic products that may affect her fetus; for such situations, an epidemiologic study of the offspring of known carriers might be useful.

Clinical management issues. Among the unresolved questions in this area are these: 1) What are the plasma levels of toxic metabolites compatible with normal growth and development? This question determines the degree to which strict dietary control can be relaxed, thus enhancing compliance. For many conditions, the answer will require better techniques for measuring accumulated toxic products. 2) What are other feasible nutritional approaches besides dietary restriction and pharma- 
cologic supplementation? Possibilities include the reduction of gastrointestinal absorption of "toxic" amino acids by means of specific ligands or colonization of the large intestine with toxindegrading recombinant organisms derived from the patient's indigenous flora. 3) What outcome variables can be used to evaluate nutritional therapy? Growth, intellect, and survival are obviously important, but more subtle effects on the quality of life of the individual and the family deserve attention.

The small number of cases of each inborn error of metabolism to appear at any one medical center and the genetic heterogeneity usually make multicenter studies necessary for evaluating therapy, but such cooperative ventures are expensive and difficult to organize. For example, carnitine is used as a dietary supplement in organic acidemias, glycine in isovaleric acidemia, and betaine in disorders of homocysteine remethylation. The rationale for the use of each of these substances appears reasonable, but controlled data supporting their clinical usefulness are lacking.

Many inborn errors of metabolism are treated with a diet comprising an amino acid mixture with very little natural protein. Over 150 such diets are commercially available for about 40 different disorders; in many cases they improve metabolic control and prolong survival. However, the poor palatability of many of these mixtures creates serious compliance problems after infancy, especially among the disorders that present with intermittent life-threatening relapses. Anorexia is often a problem, even when the patients are under metabolic control. The expertise in food technology available in academic laboratories and the vast food technology industry needs to be recruited to work on problems of diet palatability for these patients.

Possible staff responses to the recommendations in this chapter include attempts to devise new dietary therapies for orphan diseases and involve the food technology industry in efforts to improve their acceptability. Better methods for monitoring noxious intermediates in the blood might allow minimization of the amounts of special diets administered. Cooperative multicenter studies would speed the pace of therapy evaluation.

\section{CONCLUSIONS}

NICHD expenditures for nutrition research do not routinely come from earmarked funds. They reflect the funding rate of investigator-initiated applications in the nutrition area, which is not different from the overall funding rate. Occasional requests for grant applications or contract proposals can be issued by staff to stimulate work in promising neglected areas, but it is unlikely that the next few years will see much increase in this activity. To stimulate nutrition research in the future, the NICHD staff will need to function less paternally and more in a partnership with individual investigators and research societies. This could include sponsorship of workshops and meetings to stimulate interest in certain subjects, facilitation of collaborations, joint funding of projects supported in part by industry or outside foundations, and encouragement of use of available NIH programs for instrument sharing and research training. If this is successful, the next NICHD nutrition plan should find the field moving into even more exciting areas. 